\section{Statistical Motion Model Based on the Change of Feature Relationships: Human Gait-Based Recognition}

\author{
Isidro Robledo Vega, Member, IEEE, and \\ Sudeep Sarkar, Member, IEEE
}

\begin{abstract}
We offer a novel representation scheme for view-based motion analysis using just the change in the relational statistics among the detected image features, without the need for object models, perfect segmentation, or part-level tracking. We model the relational statistics using the probability that a random group of features in an image would exhibit a particular relation. To reduce the representational combinatorics of these relational distributions, we represent them in a Space of Probability Functions (SoPF), where the Euclidean distance is related to the Bhattacharya distance between probability functions. Different motion types sweep out different traces in this space. We demonstrate and evaluate the effectiveness of this representation in the context of recognizing persons from gait. In particular, on outdoor sequences 1) we demonstrate the possibility of recognizing persons from not only walking gait, but running and jogging gaits as well, 2) we study recognition robustness with respect to view-point variation, and 3) we benchmark the recognition performance on a database of 71 subjects walking on soft grass surface, where we achieve around 90 percent recognition rates in the presence of viewpoint variation.
\end{abstract}

Index Terms-Biometrics, gait recognition, relational statistics, probabilistic modeling.

\section{INTRODUCTION}

IN computer vision, the focus on identification from gait, unlike gait analysis or human motion recognition, is relatively new, except for a few demonstrations on small data sets [13], [12] in the 1990s. Over the last two years, a variety of techniques have been employed for gait-based recognition, i.e., using static body and stride parameters [4], view normalized silhouette part-based approach [19], shape symmetry [7], velocity moments [20], model-based approach [21], self-similarity plots [1], stride-length/cadence [2], silhouette width coupled with HMMs [11], and body shape [5]. The contributions of our present work lies in that it does not require part-level tracking, correspondence, alignment, part labeling, or near perfect segmentation. Most works rely on at least part-level tracking/correspondence [4], part labeling [19], require alignment of silhouettes across frames [2] or are sensitive to the quality of the silhouette [19], [7], [5], or require optic flow computations [12]. In addition, we demonstrate results on a database of 71 subjects taken outside, which is competitive with respect to the present state of art that uses five to 25 to 44 subjects, in most cases, imaged indoors.

We propose a novel strategy that emphasizes the change of the feature spatial relationships with motion, rather than the attributes of the individual features. With motion, the statistics of the relationships among the image features change. This change or nonstationarity in relational statistics is not random, but follow the motion pattern. The shape of the probability function governing the distribution of the interfeature relations that can be estimated by the normalized histogram of observed values, changes as parts of the object move. We have developed the concept of a space over these probability functions, which we refer to as the SoPF (Space of Probability Functions), to study the trend of change in their shapes.

- I. Robledo Vega is with the Technological Institute of Chihuahua, Ave. Tecnologico \#2909, Chihuahua, Chih., Mexico CP 31310.

E-mail:irobledo@itchihuahua.edu.mx.

- S. Sarkar with the Computer Science and Engineering Department, University of South Florida, 4202 E. Fowler Ave., ENB 118, Tampa, FL 33620.E-mail: sarkar@csee.usf.edu.

Manuscript received 18 Apr. 2002; revised 3 Oct. 2002; accepted 7 Mar. 2003. Recommended for acceptance by S. Sclaroff.

For information on obtaining reprints of this article, please send e-mail to: tpami@computer.org, and reference IEEECS Log Number 116376.
Distances in this space are related to the Bhattacharya distance between probability mass functions. Each motion type creates a trace in this space. By focusing on the change in relational parameters over time, we bring the dynamic aspects of the motion into fore. The use of feature attribute histograms is not new, however, the only use of relational histograms that we are aware of is by Huet and Hancock [8], who use it for image database indexing. The novelty of the present contribution lies in that we offer a strategy for incorporating dynamic aspects and use it for motion-based recognition of humans.

In the context of gait-based recognition, the specific questions that we explore in this paper are: 1) Can we identify persons from not just walking gait but jogging and running as well? 2) Is gait viewed frontal-parallel (which is the current practice) the only possibility? Can we identify humans from gait viewed at 22.5 degrees and 45 degrees? 3) Can we identify persons from a large gallery of persons walking on soft surfaces with partial occlusion of the feet?

\section{Relational Distributions}

We view an image as an assemblage of low-level features. The structure perceived in an image is determined more by the relationships among features than by the individual feature attributes. Our goal is to devise a mechanism to capture this structure so that we can use its change with time to model highlevel motion patterns. We avoid the need for feature correspondences by focusing on the statistical distribution of the relational attributes observed in the image.

Definition 1. Let 1) $\mathcal{F}=\left\{f_{1}, \cdots, f_{N}\right\}$ represent the set of $N$ features in an image, 2) $\mathbf{F}_{\mathbf{k}}$ represent a random $k$-tuple of features, and 3) the relationship among these $k$-tuple features be denoted by $R_{k}$.

Thus, 2-ary relationships between features is represented by $R_{2}$. Low-order spatial dependencies are captured by small values of $k$ and higher-order dependencies are captured by larger values of $k$.

Definition 2. Let the relationships $R_{k}$ be characterized by a set of $M$ attributes $\mathbf{A}_{\mathbf{k}}=\left\{A_{k 1}, \cdots, A_{k M}\right\}$. Then, the shape of the object can be represented by joint probability functions: $P\left(\mathbf{A}_{\mathbf{k}}=\mathbf{a}_{\mathbf{k}}\right)$, also denoted by $P\left(a_{k 1}, \cdots, a_{k M}\right)$ or $P\left(\mathbf{a}_{\mathbf{k}}\right)$, where $a_{k i}$ is the value taken by the relational attribute $A_{k i}$

We term these probabilities as the Relational Distributions. One possible interpretation of these distributions is: Given an image, if you randomly pick $k$-tuples of features, what is the probability that it will exhibit the relational attributes $\mathbf{a}_{\mathbf{k}}$ ? Or, what is $P\left(\mathbf{A}_{\mathbf{k}}=\mathbf{a}_{\mathbf{k}}\right)$ ? The representation of these relational distributions can be in parametric forms or in nonparametric, histogram, or bin-based forms. The advantage of parametric forms, such as mixture of Gaussians, is the low representational overhead. However, we have noted that these relational distributions exhibit complicated shapes that do not readily afford modeling using a combination of simple shaped distributions. So, we adopt the nonparametric histogram-based form. To reduce the size that is associated with a histogram-based representation, we propose the Space of Probability Functions that is described after we look at the following concrete example of a relational distribution.

\subsection{Moving Edge-Based Features}

We illustrate the concept of Relational Distributions by considering moving edge pixels as the features. We consider moving pixels, as they are ones most likely to belong to moving objects. To identify these edge pixels in motion, we first apply the Canny edge detector over each image frame and select only those edge pixels that fall in or within a small distance from a motion mask created by background subtraction.

Each motion edge pixel, $f_{i}$, is associated with the gradient direction, $\theta_{i}$, estimated using the Gaussian smoothed gradient. To capture the structure between two edge pixels, we use the difference in edge orientations and the distance between them as the attributes, $\left\{A_{21}, A_{22}\right\}$, of $R_{2}$. These attributes are invariant with 


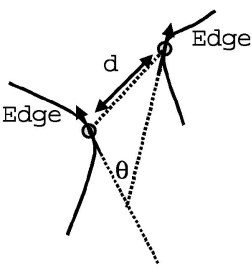

(a)

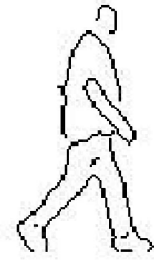

(b)

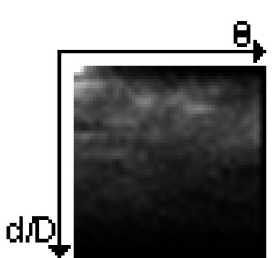

(c)

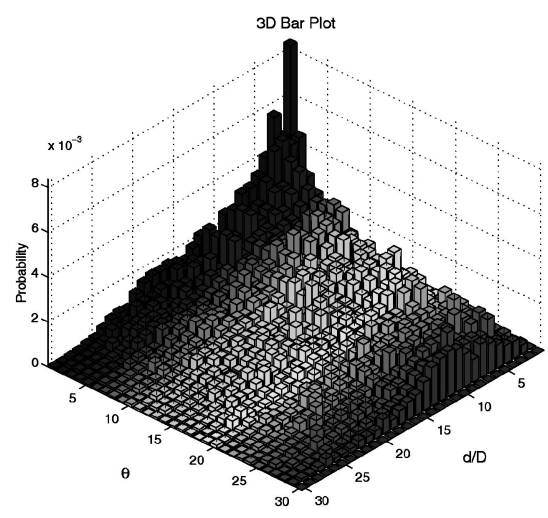

(d)

Fig. 1. Edge pixel-based 2-ary relational distribution. (a) The two attributes characterizing relationship between two edge pixels. (b) Edge pixels in an image. (c) The relational distribution $P(d / D, \theta)$, where $D$ is a scaling constant. $P(0,0)$ is the top left corner of the image. Brighter pixels denote higher probabilities. (d) The relational distribution shown as a $3 \mathrm{D}$ bar plot.

respect to image plane rotation and translation. To impart some amount of scale invariance in the representation, we normalize the distance between the pixels by a distance $(D)$ that is an estimate of the projected height of the person. We base this estimate on the straight line fit to the variation of the silhouette height with time, so as to overcome errors in height estimate in any particular frame due to segmentation errors. Fig. 1a depicts the computed attributes. And, Fig. 1c shows the $P\left(a_{21}, a_{22}\right)$ for the edge image shown in Fig. 1b. Fig. 1d shows a 3D bar plot of the probability values. Note the concentration of high values in certain regions of the probability event space. In the experiments of this paper, the 2-ary representations are 30 by 30 sized, each taking about seven seconds to compute on a $246 \mathrm{MHz}$ Sun workstation.

\section{Space of Probability Functions (SoPf)}

As the parts of an articulated object move, the relational distributions will change. Motion will introduce nonstationarity in the relational distributions. Is it possible to establish identity of the object (i.e., person) in motion? In order to enable us to answer this question, we first set up a representational scheme for these relational distributions that is easier to manipulate and is more compact than just plain histograms.

Definition 3. Let $P\left(\mathbf{a}_{\mathbf{k}}, t\right)$ represent the relational distribution at time $t$.

Definition 4. Let $\sqrt{P\left(\mathbf{a}_{\mathbf{k}}, t\right)}=\sum_{i=1}^{n} c_{i}(t) \Phi_{i}\left(\mathbf{a}_{\mathbf{k}}\right)+\mu\left(\mathbf{a}_{\mathbf{k}}\right)+\eta\left(\mathbf{a}_{\mathbf{k}}\right) d e-$ scribe the square root of each relational distribution as a linear combination of orthogonal basis functions where $\Phi_{i}\left(\mathbf{a}_{\mathbf{k}}\right)$ s are orthonormal functions, the function $\mu\left(\mathbf{a}_{\mathbf{k}}\right)$ is a mean function defined over the attribute space, and $\eta\left(\mathbf{a}_{\mathbf{k}}\right)$ is a function capturing small random noise variations with zero mean and small variance. We refer to this space as the Space of Probability Functions (SoPF).

Given a set of relational distributions, $\left\{P\left(\mathbf{a}_{\mathbf{k}}, t_{i}\right) \mid i=1, \cdots, T\right\}$, the SoPF can be arrived at by using the Karhunen-Loeve (KL) transform or, for the discrete case, by principal component analysis (PCA). The dimensions of the SoPF are given by the eigenvectors of the covariance of the square root of the given relational distributions. The variance along each dimension is proportional to the eigenvalues associated with it. In practice, we can consider the subspace spanned by a few $(N<<n)$ dominant vectors associated with the large eigenvalues. We have found that, for human motion, just $N=10$ eigenvectors are sufficient. Thus, a relational distribution can be represented using these $N$ coordinates $\left(c_{i}(t) \mathrm{s}\right)$, which is more compact representation than a normalized histogram-based representation.

We use the square root function so that we arrive at a space where the distances are not arbitrary ones but are related to the
Bhattacharya distance between the relational distributions, which is an appropriate distance measure for probability distributions.

Theorem 1. The Euclidean distance between the square root of the two relational distributions, $d_{E}\left(\sqrt{P\left(\mathbf{a}_{\mathbf{k}}, t_{1}\right)}, \sqrt{P\left(\mathbf{a}_{\mathbf{k}}, t_{2}\right)}\right)$, is monotonically related to the Bhattacharya distance between relational distribution, $d_{B}\left(P\left(\mathbf{a}_{\mathbf{k}}, t_{1}\right), P\left(\mathbf{a}_{\mathbf{k}}, t_{2}\right)\right)$, as captured by

$$
d_{E}\left(\sqrt{P\left(\mathbf{a}_{\mathbf{k}}, t_{1}\right)}, \sqrt{P\left(\mathbf{a}_{\mathbf{k}}, t_{2}\right)}\right)=2-2 e^{-d_{B}\left(P\left(\mathbf{a}_{\mathbf{k}}, t_{1}\right), P\left(\mathbf{a}_{\mathbf{k}}, t_{2}\right)\right)} .
$$

Theorem 2. In the SoPF representation, the Euclidean distance between the coordinates, $\left\{c_{i}\left(t_{1}\right)\right\}$ and $\left\{c_{i}\left(t_{2}\right)\right\}$, is monotonically related to the Bhattacharya distance between the corresponding relational distributions $P\left(\mathbf{a}_{\mathbf{k}}, t_{1}\right)$ and $P\left(\mathbf{a}_{\mathbf{k}}, t_{2}\right)$.

For the proofs of the theorems, the reader can refer to [14], [17], [15]. Note that, this use of the PCA is different from previous uses in motion tracking, e.g., [3], they use PCA over the image pixel space whereas we use it over relational probability functions. Unlike Sclaroff and Pentland [18], who use PCA for shape descriptions of deformable objects, we neither require prior shape model nor assume perfect segmentation of object from background.

\section{Distance Measures}

There are various sophisticated techniques, such as those based on hidden Markov models, dynamic Bayesian networks, and state space trajectories that can be used to model and compute distances between trajectories in the SoPF. In this paper, however, we adopt a simpler distance measure between two traces to demonstrate the viability of using the traced paths for inferring personal identity. We show in our experiments that, even with a simple distance measure, we are able to obtain good discrimination.

When comparing gait of the same type (walking or running or jogging) and with similar speed (normal), we just compute the Euclidean distance between the two traces, $\mathbf{S}_{\mathbf{1}}=\left\{\mathbf{c}^{1}\left(t_{i}\right), i=1 \cdots n\right\}$ and $\mathbf{S}_{\mathbf{2}}=\left\{\mathbf{c}^{2}\left(t_{i}\right), i=1 \cdots m\right\}$ :

$$
d_{\mathrm{un}-\text { norm }}\left(\mathbf{c}^{1}, \mathbf{c}^{2}\right)=\frac{1}{m} \sum_{t_{i}=1}^{m} \sum_{j=1}^{N}\left(c_{j}^{1}\left(t_{i}\right)-c_{j}^{2}\left(t_{i}+K\right)\right)^{2} .
$$

If the speed of motion is not controlled, i.e., slow or fast walk, or when we have to compare between walking and running gaits, we temporally normalize the two traces using dynamic time warping (DTW), allowing for just constant stretching or contraction: 


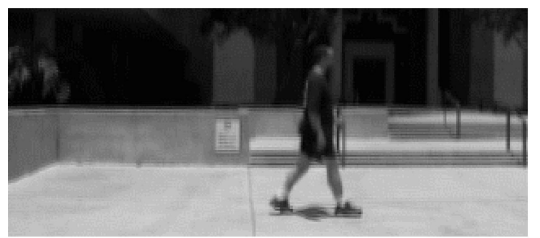

(a)

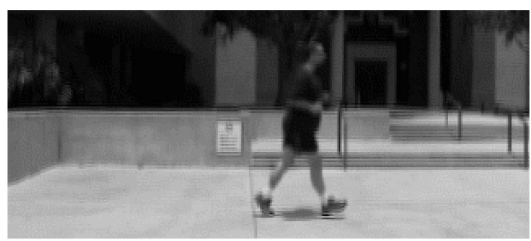

(b)

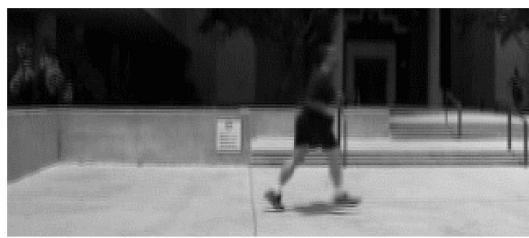

(c)

Fig. 2. Sample frames of a person (a) walking, (b) jogging, and (c) running.
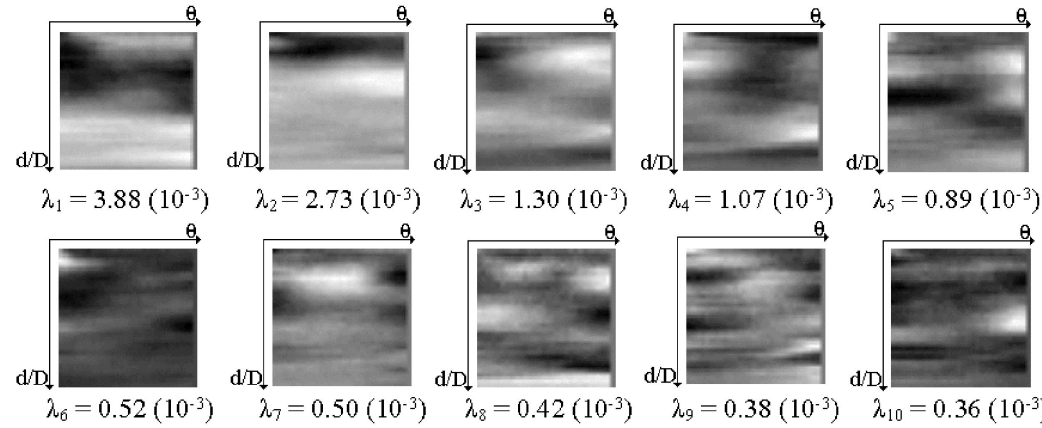

$\lambda_{5}=0.89\left(10^{-3}\right)$

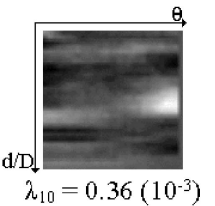

Fig. 3. Ten most dominant dimensions of the SoPF, with the corresponding eigenvalues quantifying the associated variation shown below each image. This is for the data of different motion types.

$$
d_{\text {norm }}\left(\mathbf{c}^{1}, \mathbf{c}^{2}\right)=\frac{1}{n} \sum_{t_{i}=1}^{n} \sum_{j=1}^{N}\left(\mathbf{c}_{j}^{2}\left(t_{i}\right)-\mathbf{C}_{j}^{1}\left(\frac{m}{n} t_{i}\right)\right)^{2} .
$$

The warped distance measure responds to changes in shapes of the traces over each motion cycle but does not change with the speed with which each cycle is executed. Thus, the distance between a fast walk and a slow walk would tend to be small as compared to the distance between a walk and a run cycle.

The above two distances can be directly computed from the traces if they are aligned, i.e., the starting and ending states of the two traces match. If this is not the case, then we compute a temporal correlationbased measure as follows. We partition $\mathbf{S}_{1}$ into disjoint subsequences of $K$ contiguous frames each, such that each subsequence contains roughly one cycle, denoted by $\mathbf{S}_{1}(k: k+K)=\left\{c^{1}\left(t_{k}\right), \cdots, c^{1}\left(t_{k+K}\right)\right\}$. We then compare each of these subsequences with $\mathbf{S}_{2}$ :

$$
\operatorname{Corr}\left(\mathbf{S}_{\mathbf{1}}(k: k+K), \mathbf{S}_{\mathbf{2}}\right)=\max _{l} d\left(\mathbf{S}_{\mathbf{1}}(k: k+K), \mathbf{S}_{\mathbf{2}}(l: l+K)\right) .
$$

The distance, $d$, may be the time normalized or unnormalized version of the distance between the two subsequences. The similarity is chosen to be the median value of the distance of $\mathbf{S}_{\mathbf{2}}$ with each of these $\mathbf{S}_{1}$ subsequences. This method of computing the similarity between two sequences is robust with respect to noise that distorts the motion information in a small set of contiguous frames: $\operatorname{Similarity}\left(\mathbf{S}_{\mathbf{1}}, \mathbf{S}_{\mathbf{2}}\right)=\operatorname{Median}_{k}\left(\operatorname{Corr}\left(\mathbf{S}_{\mathbf{1}}(k: k+K), \mathbf{S}_{\mathbf{2}}\right)\right)$.

\section{Identifying Persons from Walking, Jogging, AND RUNNING GaIT}

The data for the experiments described in this paper was acquired using consumer grade digital video cameras with DV compression artifacts. For this section, the image sequence database consists of 10 people performing three motion types, walking, jogging, and running, in an outdoor setting. The viewpoint is frontal-parallel and the distance from the camera is eight meters. Some example frames are shown in Fig. 2 for a person 1) walking, 2) jogging, and 3) running. From these images, we can see that the inclination of the body is different, specially the upper body. Arms position and movement are also different. Each person performed these three different motion types in two different directions, left-to-right and right-toleft. This gives us six different types of sequences (Walking-Left, Walking-Right, Jogging-Left, Jogging-Right, Running-Left, Running-Right) for each person, resulting in a total of 60 sequences.

\subsection{Analysis of Covariates}

The three covariates present in the 10 person database are: motion type, walking direction, and the identity of the person. In this section, we quantify the strength of the variations in gait due to these covariates. For our analysis, from each of the 60 sequences, we manually extracted two motion cycles: One was used to build the SoPF (training set) and the other was used for analysis (testing set). The dimensions of the trained SoPF are shown in Fig. 3 as gray-level images. Variation of distances seems to be important for the top eigenvectors and the orientation variations are emphasized by later eigenvectors.

We computed the time-normalized distances $\left(d_{\text {norm }}\right)$ between each pair of the 60 training and 60 testing sequence cycles. We then used analysis of variance (ANOVA [16]) to study the effect of person, motion type, and direction of motion on the computed distance. Each covariate can have two possible values: same or different, i.e., same person or different persons, same motion type or different motion types, and same motion direction and different motion directions. The computed ANOVA table is shown in Table 1, from which we can see that differences due to the subject is, by far, the largest source of variation as compared to motion type or direction. The subject effect could be a combination of the identity of the person and his/her clothing. We investigate this issue in some depth later.

TABLE 1

ANOVA Table with Results for Different Motion Types Experiments

\begin{tabular}{|c|c|c|c|c|}
\hline Source & DF & SS & F-value & P-value \\
\hline \hline Person & 1 & 793.92 & 114.22 & $<0.0001$ \\
\hline Angle & 1 & 9.53 & 1.37 & 0.2419 \\
\hline Direction & 1 & 12.06 & 1.74 & 0.1879 \\
\hline
\end{tabular}


TABLE 2

Number of People Correctly Identified for Different Motion Types Experiments

\begin{tabular}{|c||c||c||c|}
\hline \multirow{2}{*}{ At } & \multicolumn{3}{c|}{ Experiment } \\
\cline { 2 - 4 } Rank & Gallery: Walking Left & Gallery: Jogging Left & Gallery: Running Left \\
& Probe: Walking Right & Probe: Jogging Right & Probe: Running Right \\
\hline \hline 1 & 10 of 10 & 10 of 10 & 8 of 10 \\
\hline 2 & 10 of 10 & 10 of 10 & 9 of 10 \\
\hline
\end{tabular}

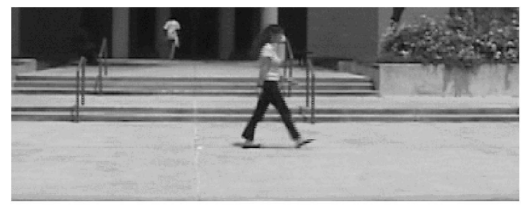

(a)

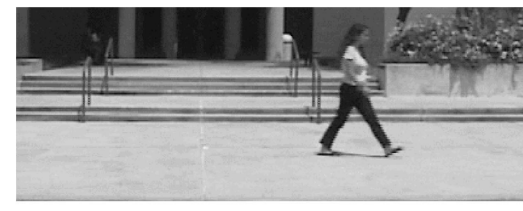

(b)

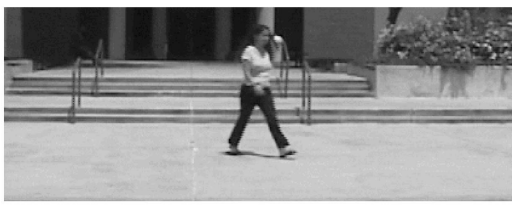

(c)

Fig. 4. Sample frames from the same person walking (a) frontal-parallel, (b) 22.5 degrees, and (c) 45 degrees with respect to the image plane.

\subsection{Gait-Based Recognition Experiments}

Given that the subject is the largest source of variation in the distances out of the three factors, it is natural to ask what kind of recognition rates we can get based on gait, be it walking, jogging, or running. We conducted three gait recognition experiments based on walking, jogging, and running gaits. For each experiment, we separated the sequences with the corresponding motion type into gallery and probe sets, adopting the defacto standard FERET (Face Recognition Technology) evaluation methodology [9]. One cycle from each sequence with the person going left formed the gallery set and one cycle from each sequence with the person going right formed the probe sets. Basically, we are using the left profile of the person as gallery and the right profile as probe. The specific gallery and probe sets for each experiment are listed in the second row of Table 2. The gallery set of images was also the training set used to form the SoPF.

For each probe, we compute its distance from all the gallery images. If the identity of the gallery image with the smallest distance to the probe matches the identity of the probe, then we have successful identification. Table 2 shows the results of the recognition experiments. We have perfect identification at rank 1 for walking and jogging gaits. The rate for running gait is also not too bad: 8 out of 10 at rank 1 and 9 out of 10 at rank 2. It is interesting to note that Yam et al. [21] also observed in their experiments with image data of persons on a treadmill that running gait is also a potential source of biometric.

\section{WALKING Gait-BASEd IDENTIFICATION UNDER Different VieW ANGLes}

In this section, we investigate the relationship of the achieved recognition rates with viewing angle. For this, we imaged 20 persons walking frontal-parallel, 22.5 degrees, and 45 degrees with respect to the image plane. The distance from the camera to the frontal-parallel path was 12 meters. Each person walks each of the three slanted paths in two different directions, left-to-right and right-to-left, resulting in six sequences per person at 0 degrees (frontal-parallel) going left (0L), 0 degrees (frontal-parallel) going right (0R), 22.5 degrees going left (22L), 22.5 degrees going right (22R), 45 degrees going left (45L), and 45 degrees going right (45R). The abbreviations in parentheses will be used in the following discussion to refer to these conditions. Fig. 4 shows three sample frames from the same person walking the three differently angled paths. The frame size is $280 \times 130$. We stop at 45 degrees because for view angles greater than this, leg and arm motion becomes more difficult to be captured in 2D projected images and other aspects like body shape (height and weight) become more important than gait information.

\subsection{Analysis of Covariates}

The three covariates present in the database for this experiment are: walking direction, angle of motion path, and the person. We quantify the strength of effect of these factors on the variations in the distance values computed between two cycles from each of the 120 sequences $(20$ persons $\times 3$ covariates $\times 2$ conditions per covariates). One cycle from each of the 120 sequences form the training set of images that is used to construct the SoPF. As before, we quantify the effect of the covariates on the distances using ANOVA, whose output is shown in Table 3. We see that the person is the largest and most significant source of variation. In fact, as the F-values suggest, the variation due to the persons is at least three orders of magnitude larger than due to angle or walking direction.

\subsection{Gait-Based Recognition Experiments}

Given that the person is the largest source of gait variation; as measured in the SoPF, how do the recognition rates vary with view angle? To answer this, we separated our database into five sets of gallery and probe combinations. The going-left sequences form the galleries and the going-right sequences form the probes. The training set of images used to create the SoPF, consists of the union of the gallery sets. In the first set of experiments, we study if recognition can be possible from views other than frontal-parallel ones. In the second set of experiments, we study how recognition varies with change in view angle. Table 4 lists the identification rates at ranks 1 and 2 for these two sets of experiments. From the first set of experiments, we see that when the gallery and probes

TABLE 3

ANOVA Table with Results for Different View Angle Experiments

\begin{tabular}{|c|c|c|c|c|}
\hline Source & DF & SS & F-value & P-value \\
\hline \hline Person & 1 & 4624.33 & 1208.97 & $<0.0001$ \\
\hline Angle & 1 & 0.74 & 0.19 & 0.6604 \\
\hline Direction & 1 & 4.51 & 1.18 & 0.2775 \\
\hline
\end{tabular}


TABLE 4

Identification Rates for Experiments Studying (a) Possibility of Recognition from Different View Points, but the Gallery and Probes Are from the Same Viewpoint, and (b) Fall of Recognition Rates as the View Angle of the Probe Differ from the Gallery

\begin{tabular}{|c||c||c||c|}
\hline $\begin{array}{c}\text { Gallery } \\
\text { Probe }\end{array}$ & OR & $22 \mathrm{~L}$ & $45 \mathrm{~L}$ \\
$22 \mathrm{R}$ & $45 \mathrm{R}$ \\
\hline \hline Rank 1 & $80 \%$ & $75 \%$ & $80 \%$ \\
\hline Rank 2 & $90 \%$ & $95 \%$ & $85 \%$ \\
\hline
\end{tabular}

\begin{tabular}{|c||c||c||c|}
\hline $\begin{array}{c}\text { Gallery } \\
\text { Probe }\end{array}$ & OL & OL & OL \\
\hline \hline Rank 1 & $80 \%$ & $75 \%$ & $55 \%$ \\
\hline Rank 2 & $90 \%$ & $80 \%$ & $80 \%$ \\
\hline
\end{tabular}

(a)

(b)

are from the same view angle, the rates are similar. We can conclude that gait-based recognition is possible from nonfrontal-parallel views, such as those viewed at 22.5 degrees or 45 degrees. From the second set of experiments, where we progressively varies the view angle of the probe with respect to the gallery, we see that the identification rate drops to 75 percent when the probe is from 22.5 degrees viewpoint. But, the fall is drastic, to 55 percent, with a 45 degrees viewpoint probe set. Thus, it appears that the gait-based recognition using the SoPF framework seems to be robust with respect to viewpoint change up to 22.5 degrees.

One might argue that, on a small data set, one should get near 100 percent identification rates. To this, we point out the complexity of the outdoor imaging conditions in the data set and the fact that we have a clear separation of train and test sets; we use the left profile for training (or as gallery) and try to identify people from their right profiles (the probe sets). Thus, the recognition rates also reflect the inherent variation in gait due to opposite profile viewpoints in addition to any other factor that might be different between the probe and gallery sets in each of the experiments.

\section{Walking Gait-Based Recognition on SOFT SURFACE}

In this section, we present results on a larger data set of 71 subsets on a soft surface, i.e., grass that is usually not considered. This data set is a subset of the recently formulated HumanID Gait Challenge problem [10]. Subject demographics were as follows: 75 percent male, age: 19 to 54 years, height: 1.47 to 1.91 meters, and weight: 43.1 to 122.6 kilograms. Subjects walked five to six laps around an elliptical path in front of two cameras verged at about 30 degrees and about 16-18 meters away from the subject. So, as to factor out gait changes due to the subjects knowing that they are being video taped, we considered only the last lap, from which only the back (farthest) portion of the lap was considered. This contained about 280 and 350 frames per subject containing over four to five gait cycles. Fig. 5 shows two sample views.

We use this database to investigate how well the SoPF representation performs on a large database and on a soft surface that occludes part of the feet. In a first experiment, we used sequences from the right camera (Right View) as our training/ Gallery set to build the SoPF and the sequences from the left camera (Left View) as the Probe. Then, in a second experiment, we repeated the study by reversing the Gallery and Probe sets. In a third experiment, we randomly mixed the sequences from the Right View with those from the Left View to generate the Gallery and Probe sets with the purpose of showing the bias effects that could be produced by the partitions generated in the previous experiments. For these experiments, we used the sequence correlation strategy to measure distances between sequences containing multiple gait cycles that was discussed earlier. Table 5 lists the obtained identification rates of 90 percent, 89 percent, and 82 percent that are comparable with previous results. These experiments also demonstrate that manual segmentation of gait cycles and part tracking are not necessary.

In an attempt to shed some light on how much clothing/body shape impacts the recognition based on SoPF, we considered recognition from a single frame. We selected the frame when the heels are together that is the frame with the least gait related information. The identification rate was just 10 percent that is a considerable drop compared with rates shown in Table 5. This suggests that the representation is not latching onto clothing/body shape related factors in a significant manner.

\section{Conclusions}

We presented a statistical framework for motion analysis that tracks the variation of nonstationarity in the distributions of relations among image features in individual frames, which is facilitated using the concept of a Space of Probability Functions (SoPF). Among the attractive features of this approach are 1) no feature-level tracking or correspondence is necessary, 2) there is no need for explicit object shape models, and 3) movement between frames need not be in the order of one or two pixels. We demonstrated and evaluated the effectiveness of this representation for the task of gait-based identification. Qualitative conclusions that can be drawn from these studies are:

1. The subject is a far greater source of gait variation than viewpoint, motion types, or direction of motion.

2. It is possible to recognize persons from jogging and running gaits and not just from walking gait.

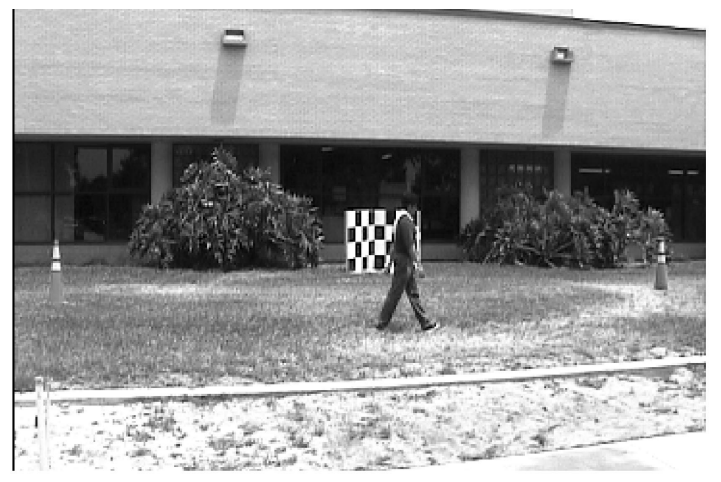

(a)

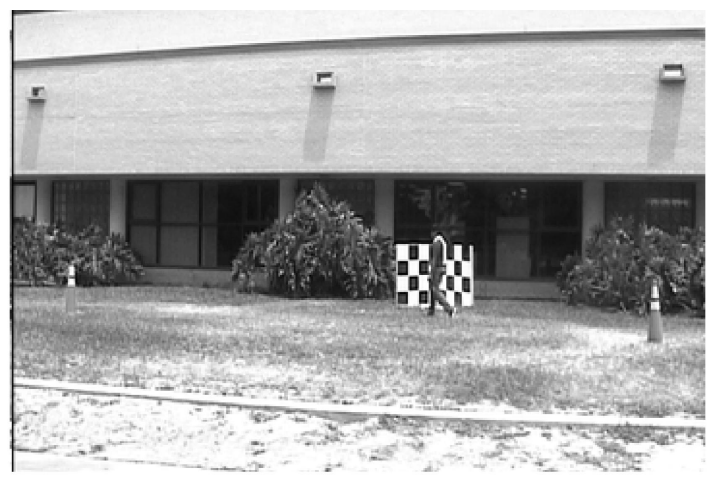

(b)

Fig. 5. Frames from (a) the left camera and (b) the right camera. 
TABLE 5

Identification Rates When Reversing Gallery and Probe

\begin{tabular}{|c||c|c||c|c|c|c|}
\hline \multicolumn{1}{|c||}{} & \multicolumn{1}{c||}{} & \multicolumn{4}{c|}{ At rank } \\
\cline { 5 - 7 } Exp. & Gallery & Probe & 1 & 2 & 3 & 4 \\
\hline \hline 1 & Right View & Left View & $90 \%$ & $96 \%$ & $97 \%$ & $99 \%$ \\
\hline 2 & Left View & Right View & $89 \%$ & $97 \%$ & $99 \%$ & $99 \%$ \\
\hline 3 & Randomly Picked & Randomly Picked & $82 \%$ & $91 \%$ & $93 \%$ & $96 \%$ \\
\hline
\end{tabular}

3. Gait-based recognition need not be restricted to frontalparallel views; walking gaits viewed from 22.5 degrees and 45 degrees also results in similar recognition as that from frontal-parallel views.

4. We can get up to 80 to 90 percent recognition in the presence of just viewpoint variation.

Experiments also suggest that body shape and clothing do not seem to be contributing to the recognition rates.

Future studies will involve more detailed analyses of gait recognition problem along the lines charted in the HumanID Gait Challenge problem [10]. The effect of walking speed on gait is also an important problem that is difficult to normalize. Most systems, including ours, use some variation of temporal warping to handle varying speeds. Some train using samples at various speeds [2]. Some suggest the use normalized static parameters computed from cadence, stride length, height, or limb lengths [6], [4], [2]. However, recognition with these parameters extracted from real images is usually low. Another relationship of interest is the effect of image resolution on recognition. We expect the rate to drop with resolution [2]. In general, systems that use global similarity measures, such as ours or [1], should work well with lowresolution images, but systematic study is still needed.

\section{ACKNOWLEDGMENTS}

This research was supported by funds from US National Science Foundation grant EIA 0130768 and DARPA HumanID program under contract AFOSR-F49620-00-1-00388. Dr. Robledo-Vega acknowledges CONACYT-SEP-Mexico for supporting his $\mathrm{PhD}$ studies.

\section{REFERENCES}

[1] C. BenAbdelkader, R. Cutler, and L. Davis, "Motion-Based Recognition of People in Eigengait Space," Proc. Int'l Conf. Automatic Face and Gesture Recognition, pp. 267-272, 2002.

[2] C. BenAbdelkader, R. Cutler, and L. Davis, "Stride and Cadence as a Biometric in Automatic Person Identification and Verification," Proc. Int'l Conf. Automatic Face and Gesture Recognition, pp. 372-377, 2002.

[3] M. Black and A. Jepson, "EigenTracking: Robust Matching and Tracking of Articulated Objects Using View-Based Representation," Proc. European Conf. Computer Vision, pp. 329-342, 1996.

[4] A. Bobick and A. Johnsson, "Gait Recognition Using Static, ActivitySpecific Parameters," Computer Vision and Pattern Recognition, pp. 423-430, 2001.

[5] R. Collins, R. Gross, and J. Shi, "Silhouette-Based Human Identification from Body Shape and Gait," Proc. Int'l Conf. Automatic Face and Gesture Recognition, pp. 366-371, 2002.

[6] J. Davis, "Visual Categorization of Children and Adult Walking Styles," Proc. Int'l Conf. Audio- and Video-Based Biometric Person Authentication, pp. 295-300. 2001.

[7] J. Hayfron-Acquah, M. Nixon, and J. Carter, "Automatic Gait Recognition by Symmetry Analysis," Proc. Third Int'l Conf. Audio- and Video-Based Biometric Person Authentication, pp. 272-277, 2001.

[8] A. Huet and E. Hancock, "Line Pattern Retrieval Using Relational Histograms," IEEE Trans. Pattern Analysis and Machine Intelligence, vol. 21, no. 12, pp. 1363-1370, Dec. 1999.
[9] P.J. Phillips, H. Moon, S. Rizvi, and P. Rauss, "The FERET Evaluation Methodology for Face-Recognition Algorithms," IEEE Trans. Pattern Analysis and Machine Intelligence, vol. 22, no. 10, pp. 1090-1104, Oct. 2000.

[10] P.J. Phillips, S. Sarkar, I. Robledo, P. Grother, and K. Bowyer, "The Gait Identification Challenge Problem: Data Sets and Baseline Algorithm," Proc. Int'l Conf. Pattern Recognition, pp. 385-388, 2002.

[11] A. Kale, A. Rajagopalan, N. Cuntoor, and V. Kruger, "Human Identification Using Gait," Proc. Int'l Conf. Automatic Face and Gesture Recognition, pp. 336341, 2002.

[12] J. Little and J. Boyd, "Recognizing People by Their Gait: The Shape of Motion," Videre, vol. 1, no. 2, pp. 1-33, 1998.

[13] S.A. Niyogi and E.H. Adelson, "Analyzing and Recognizing Walking Figures in XYT," Computer Vision and Pattern Recognition, 1994.

[14] I. Robledo Vega, "Motion Model Based on Statistics of Feature Relations: Human Identification from Gait," PhD dissertation, Univ. of South Florida, Aug. 2002.

[15] I. Robledo Vega and S. Sarkar, "Experiments on Gait Analysis by Exploiting Nonstationarity in the Distribution of Feature Relationships," Proc. Int'l Conf. Pattern Recognition, pp. 1-4, 2002.

[16] T. Sanocki, Student-Friendly Statistics. Prentice Hall, 2000.

[17] S. Sarkar and I. Robledo Vega, "Discrimination of Motion Based on Traces in the Space of Probability Functions Over Feature Relations," Computer Vision and Pattern Recognition, pp. 976-983, 2001.

[18] S. Sclaroff and A. Pentland, "Modal Matching for Correspondence and Recognition," Trans. Pattern Analysis and Machine Intelligence, vol. 17, no. 6, pp. 545-561, June 1995.

[19] G. Shakhnarovich, L. Lee, and T. Darrell, "Integrated Face and Gait Recognition from Multiple Views," Computer Vision and Pattern Recognition, pp. 439-446, 2001.

[20] J. Shutler, M. Nixon, and C. Carter, "Statistical Gait Description via Temporal Moments," Proc. Fourth IEEE Southwest Symp. Image Analysis and Interpretation, pp. 291-295, 2000.

[21] C. Yam, M. Nixon, and J. Carter, "Extended Model-Based Automatic Gait Recognition of Walking and Running," Proc. Third Int'l Conf. Audio- and Video-Based Biometric Person Authentication, pp. 278-283, 2001.

$\triangleright$ For more information on this or any other computing topic, please visit our Digital Library at http://computer.org/publications/dlib. 